\title{
Maser Mapping of Red Supergiants and the Onset of Bipolar Outflow
}

\author{
ANITA M. S. RICHARDS ${ }^{1}$, JEREMY A. YATES ${ }^{2}$, \\ and R. JAMES COHEN ${ }^{1}$
}

1 Jodrell Bank, University of Manchester, Cheshire, U.K.

${ }^{2}$ School of Chemistry, University of Bristol, Bristol, U.K.

Maser emission from $\mathrm{SiO}, \mathrm{H}_{2} \mathrm{O}$ and $\mathrm{OH}$ is found in oxygen-rich red giant circumstellar envelopes at successively larger distances from the star. $\mathrm{H}_{2} \mathrm{O}$ emission at $22 \mathrm{GHz}$ has been mapped with MERLIN (the Multi Element Radio Linked Interferometer, http://www.jb.man.ac.uk/merlin/MERLIN.html) with a beamsize of 8 mas. Typically the $22 \mathrm{GHz}$ emitting region is seen to be a thick expanding shell 100 mas across. Small-scale clumpiness is revealed. The shell is resolved into discrete features of velocity extent 1 to $3 \mathrm{~km} \mathrm{~s}^{-1}$ and spatial extent 2 to 20 mas. Using this $3-\mathrm{D}$ datacube, constraints are placed on the physical conditions such as kinetic temperature, density and local radiation field. These observations show that the size of the region increases with stellar mass-loss rate and that the mass outflow is undergoing acceleration. The outflow is gravitationally bound to the star at the inner radius of the $\mathrm{H}_{2} \mathrm{O}$ masing region, but unbound at its outer radius (Chapman \& Cohen 1986, $M N R A S, 230,415$; Yates \& Cohen 1994, MNRAS, 270, 529).

In NML Cyg (mass $\sim 50 M_{\odot}$ ), proper motion has been detected in two isolated features outside the rest of the $\mathrm{H}_{2} \mathrm{O}$ maser shell which are symmetric in position and velocity with respect to the stellar position (Richards, Yates \& Cohen 1996, MNRAS, 282, 665). The increase in separation over 9 years corresponds to a transverse velocity of $19 \pm 6 \mathrm{~km} \mathrm{~s}^{-1}$ at a distance of $2 \mathrm{kpc}$. This is consistent with the observed radial-velocity range of the $22 \mathrm{GHz}$ emission. $\mathrm{OH}$ and dust observations show a similar axis of symmetry, and this may be a sign of the onset of bipolar outflow. Axisymmetric structures are often seen in supernova remnants, and also in the planetary nebulae which originate from $\sim 1 M_{\odot}$ stars. Results are also given for other supergiant stars currently being observed.

Papers relating to this work will be available by ftp; those interested should write to amsr@jb.man.ac.uk for details. 